\title{
Evolução Dos Conceitos Da Física Quântica: Uma Descrição Histórica A Partir Da Evolução Da Termodinâmica E Do Eletromagnetismo
}

\author{
Evolution Of The Concepts Of Quantum Physics: A Historical \\ Description From The Evolution Of Thermodynamics And \\ Electromagnetism
}

Luciano Galdino1, João Carlos Lopes Fernandes²

\begin{abstract}
1. Professor de Física e Elementos de Máquina na Faculdade ENIAC. Mestre em Ciências Exatas e da Terra na área de Física Nuclear pela USP, especializado em Física pela USP e Licenciado em Matemática pela UNG. E-mail: lucianogaldino1@yahoo.com.br.

2. Professor pesquisador na Faculdade ENIAC, professor associado do IMT e coordenador na Fatec São Caetano do Sul. Doutor em Engenharia biomédica pela UMC, Mestre em engenharia da computação IPT/USP, e Licenciado em Ciência da computação pela USCS. E-mail: joao.carlos@eniac.com.br.
\end{abstract}

\section{Resumo}

Este artigo aborda o desenvolvimento da Física Quântica através de uma evolução cronológica e aponta os principais físicos que contribuíram com as suas teorias e também com os experimentos que validaram ou não essas teorias. A termodinâmica e o eletromagnetismo são duas grandes áreas da física que tiveram um papel decisivo para o surgimento e também para o desenvolvimento da Física Quântica e por esse motivo é mencionado neste artigo uma descrição breve de suas principais descobertas, destacando as teorias que culminaram como contribuição à Física Quântica. O tratamento matemático exposto se resume na apresentação das principais equações, isto é, não foi exposto o tratamento matemático detalhado e avançado, pois o foco do trabalho é de apresentar a evolução dos conceitos. É destacado também o desenvolvimento simplificado da estrutura atômica por ter sua evolução concomitante com a evolução da Física Quântica.

Palavras-Chave: Física. Quântica. Termodinâmica. Eletromagnetismo. 


\begin{abstract}
This article discusses the development of quantum physics through a chronological development and points out the major physicists who contributed with their theories and the experiments that validated or not these theories. Thermodynamics and Electromagnetism are two major areas of physics that had a decisive role to the appearance and for the development of quantum physics and for that reason is mentioned in this article a brief description of its main findings, bighlighting the theories, which culminated as a contribution to quantum physics. The mathematical treatment above is summarized in the presentation of the main equations, i.e. was not exposed the detailed and advanced mathematical treatment, because the focus of the job is to present the evolution of the concepts. Is highlighted also the simplified development atomic structure by having their development concurrent with the development of quantum physics.
\end{abstract}

Keywords: Physics. Quantum. Thermodynamics. Electromagnetism.

\section{Introdução}

A física quântica é uma das principais áreas da atualidade da física, pois sua teoria se aplica em diversos âmbitos da ciência e desperta dúvidas e curiosidades, pois possui conceitos intrigantes e diferenciados, mesmo nas pessoas não ligadas à ciência.

O objetivo desse artigo é explicar a evolução da Física Quântica, mostrando em ordem cronológica e destacando os principais físicos envolvidos nesse processo.

Uma breve evolução da termodinâmica e do eletromagnetismo é apresentada, indicando as teorias e resultados experimentais que culminaram no nascimento da Física Quântica, pois os resultados experimentais não estavam de acordo com a teoria Física da época (Física clássica).

Os autores descrevem essa evolução através de conceitos físicos de fácil entendimento e de forma qualitativa, isto é, discutindo os conceitos sem a explanação de uma matemática mais sofisticada, apontando apenas as principais equações obtidas, assim, para o entendimento desse artigo, não há necessidade de possuir um profundo conhecimento Físico e nem Matemático.

O artigo explora também o desenvolvimento da estrutura atômica e a Física Quântica, pois é justamente no universo atômico que as leis da física clássica falham e que as leis da Física Quântica apontaram concordância com os resultados experimentais.

Dentro do estudo da Física Quântica existe uma separação que praticamente é em função da aplicação de uma matemática mais avançada para explicar a teoria da dualidade ondapartícula, sendo que até por volta de 1925 tinha-se a chamada antiga teoria quântica e a partir dessa data a nova teórica quântica denominada mecânica quântica ou mecânica ondulatória que teve como base a famosa equação de Schrödinger. 
O artigo também apresenta os conflitos entre as teorias e destaca os principais experimentos realizados recentemente para ressaltar que ainda existe a necessidade de desenvolvimentos de experimentos mais precisos que comprovem as teorias propostas para que a Física Quântica tenha uma consolidação por completo.

\section{A termodinâmica apontando para a necessidade de uma nova física.}

Em 1760, o escocês Joseph Black (1728 1799) realizou a melhor definição de calor e apresentou uma distinção clara com relação à temperatura, enunciando calor como uma troca de energia entre os corpos e temperatura como o grau de agitação molecular. Considerou o calor como um fluido ponderável e indestrutível e introduziu os conceitos de capacidade térmica e calor latente dos corpos. É considerado o fundador da termometria (PIRES, 2008).

Após a metade do século XIX, mais precisamente em 1868, o britânico James Clerk Maxwell (1831 - 1879) e o austríaco Ludwig Eduard Boltzmann (1844 - 1906) desenvolveram, paralelamente, a teoria cinética dos gases, baseada nas leis de Newton, isto é, seguindo a mecânica newtoniana, dando origem à famosa distribuição de Maxwell-Boltzmann (GRIBBIN, 2002).

O Inglês Willian Thompson (1824 - 1907), também conhecido como barão Kelvin, e o alemão Rudolf Julius Emanuel Clausius (1822 1888), estabeleceram a segunda lei da termodinâmica enunciando que o calor flui de um corpo quente para um corpo frio espontaneamente, mas nunca ao contrário. Assim foi possível também conceituar entropia ${ }^{1}$ relacionando-a com a segunda lei da termodinâmica.

Em 1859, o alemão Gustav Robert Kirchhoff (1824 - 1887) iniciou estudos sobre a radiação do corpo negro ${ }^{2}$ procurando uma relação entre temperatura de um corpo que emitia luz e as propriedades da radiação emitida e provar que o fluxo emitido é independente do material. O austro-esloveno Josef Stefan (1835 - 1893), em 1879, concluiu que o fluxo emitido (申) era proporcional à quarta potência da temperatura (equação 1). Em 1884 Boltzmann chegou ao mesmo resultado estudando a emissão e absorção da radiação em uma cavidade e utilizando conceitos da termodinâmica clássica e do eletromagnetismo. Essa relação foi confirmada por experimentos

${ }^{1}$ Conceitualmente entropia é definida como o grau de desordem num sistema. Se o sistema for fechado e irreversível a entropia sempre aumenta e não obedece a uma lei de conservação. Matematicamente pode ser definida como o produto da constante de Boltzmann (k) pelo logaritmo do número de estados acessíveis do sistema com uma dada energia (W), $(S=k \log W)$, (PIRES, 2008). Segundo Halliday, Resnick e Walker (2002) a variação da entropia $(\Delta S)$ é definida como a integral da razão entre a quantidade de calor infinitesimal (dQ) e temperatura (T) $\left(\Delta S=\int_{i}^{f} \frac{d Q}{T}\right)$, sendo que para sistemas fechados e reversíveis a entropia permanece constante.

${ }^{2}$ Corpo negro ideal é um corpo que absorve toda a radiação incidente (TIPLER e LLEWELLYN, 2001). 
em 1897 e foi chamada de Lei de StefanBoltzmann (MARTINS; ROSA, 2014).

$$
\phi=K T^{4}
$$

Onde $\mathrm{K}=5,6705 \times 10^{-8} \mathrm{~W} /\left(\mathrm{m}^{2} \mathrm{~K}^{4}\right)$.

Em 1893, o alemão Wilhelm Jan Wien (1864 - 1928) analisando o comportamento da radiação em um cilindro com paredes refletoras concluiu que o comprimento de onda para o qual a radiação é máxima varia inversamente à temperatura, isto é, o produto da temperatura (T) pelo comprimento de onda máximo $\left(\lambda_{m}\right)$ é uma constante dada pela equação 2 e conhecida como a lei de deslocamento de Wien (TIPLER; LLEWELLYN, 2001).

$$
\lambda_{m} \cdot T=2,898 \times 10^{-3} \mathrm{mK}
$$

Martins e Rosa (2014) afirmam que para satisfazer ao mesmo tempo a lei do deslocamento e a lei de Stefan-Boltzmann, Wien elaborou outra lei (Lei de Wien) em 1896 (equação 3) tomando como base a distribuição de velocidades de moléculas de um gás perfeito que foi confirmada experimentalmente em 1897 pelos alemães Friedrich Paschen (1865 - 1947) e H. Wanner, mas somente para baixa temperatura $(\mathrm{T})$ e alta frequência luminosa (f).

$$
d u=\alpha f^{3} e^{-\left(\frac{\beta f}{T}\right)} d f
$$

Onde u é a distribuição de energia no espectro da radiação de corpo negro.

Segundo Bergia (2012) os alemães Otto Richard Lummer (1860 - 1925) e Ernst Pringsheim (1859 - 1917) demonstraram em 1899 que a lei de Wien não era válida para baixas frequências.

Em 1900, os ingleses John Willian Strutt, mais conhecido como Lord Rayleigh (1842 1919) e Sir James Hopwood Jeans (1877 1946) elaboraram uma nova lei do espectro do corpo negro (Lei de Rayleigh-Jeans) baseado na equipartição da energia e ondas estacionárias formadas em cavidades com uma pequena abertura (equação 4). Essas cavidades simulam um corpo negro porque a radiação que entra pela pequena abertura na cavidade possui uma probabilidade muito pequena de sair antes de ser absorvida pelas reflexões nas paredes das cavidades (TIPLER; LLEWELLYN, 2001).

$$
u(\lambda)=8 \pi k T \lambda^{-4}
$$

Analisando a lei de Rayleigh-Jeans (equação 4), observa-se que para comprimentos de onda grandes a lei está de acordo com os resultados experimentais, mas para comprimentos de onda muito pequenos (tendendo a zero) ela prevê uma densidade de energia tendendo ao infinito ficando em desacordo com os resultados 
experimentais que indicam uma tendência a zero, isto é, os conceitos físicos da época (física clássica) relacionados à radiação apresentavam discordância com resultados experimentais. Essa discrepância ficou conhecida como catástrofe do ultravioleta e deixou claro que algum conceito físico deveria ser modificado.

\section{A evolução do estudo da luz e do eletromagnetismo como base para a nova física.}

A partir do final do século XVII os estudos sobre a natureza da luz tomou uma dimensão maior, isto é, procurava-se definir como ela se propagava e como era gerada. Existiam duas teorias: a corpuscular e a ondulatória. $\mathrm{O}$ inglês Isaac Newton (1643 - 1727), que publicou uma grande obra sobre ótica em 1704 e a segunda edição em 1717, não deixava claro qual teoria defendia, pois em suas obras havia a combinação de ambas com uma tendência à teoria corpuscular. Já o holandês Christiaan Huygens (1629 - 1695) propunha uma teoria ondulatória para luz, tanto que é considerado como o fundador da teoria ondulatória por ter publicado em 1690 a sua obra "Tratado sobre a luz” (NUSSENZVEIG, 2002).

Em 1800, o britânico Thomas Young (1773 - 1829), mostrou experimentalmente que a luz sofria interferência, demonstrando assim que a teoria de Huygens estava correta, isto é, a luz se propagava em forma de onda, pois já sabia na época que interferência era um fenômeno óptico. Em 1809, o francês François Jean
Dominique Arago (1786 - 1853) demonstrou o fenômeno da polarização da luz e provou a existência de vibrações transversais, reforçando ainda mais o caráter ondulatório da luz (PIRES, 2008).

Nussenzveig (2002) destaca que assim como Thomas Young, o francês Augustin Jean Fresnel (1788 - 1827) teve um papel importantíssimo no desenvolvimento da ótica, principalmente com relação aos fenômenos de interferência e difração.

Até o momento, pelos estudos desenvolvidos em óptica, tanto teóricos como experimentais, não havia nenhuma discussão, a luz possuía caráter ondulatório, mas não se sabia ainda que a luz era uma onda eletromagnética.

Há relatos que na Grécia antiga já se sabia eletrizar os corpos tanto por atrito como por indução, mas somente em 1737 que o francês Charles François de Cisternay Du Fay (1698 1739) constatou que na eletrização por atrito os corpos envolvidos ficavam com eletrizações diferentes (ele realizou experimentos atritando o âmbar, borracha ou qualquer material resinoso com vidro ou mica), isto é, ocorriam atrações e repulsões entre os corpos atritados (nascimento do conceito de carga positiva e carga negativa). Em 1729, o inglês Stephen Gray (1666 - 1736) demonstrou que a eletrização produzida nos corpos poderia se deslocar para outros corpos e que isso somente ocorreria devido à existência de um fluido elétrico. $\mathrm{O}$ estadunidense Benjamim Franklin 
(1706 - 1790), em meados do século XVIII, enunciou que a eletricidade estava presente em toda matéria, através de resultados obtidos por experimentos com a garrafa de Leyden (dispositivo capaz de armazenar eletricidade) e enunciou a lei da conservação da carga elétrica. Em 1767, o britânico Joseph Priestley (1733 1804) propôs que a força elétrica entre os corpos devia obedecer a uma lei do tipo do inverso do quadrado da distância (PIRES, 2008).

Halliday, Resnick e Walker (2003) afirmam que somente em 1785, o francês Charles Augustin Coulomb (1736 - 1806), depois de vários experimentos, formalizou a equação da força elétrica (lei de Coulomb) através da equação 5 .

$$
\vec{F}_{e}=\frac{K Q q}{\vec{r}^{2}}
$$

Onde:

$\mathrm{Fe}=$ Força elétrica;

$\mathrm{K}=$ Constante elétrica;

Q e $\mathrm{q}=$ cargas elétricas envolvidas;

$r=$ distância entre as cargas elétricas.

Segundo Pires (2008) o dinamarquês Hans Christian Oersted (1777 - 1851) iniciou estudos relacionados com a ação da eletricidade em uma agulha imantada em 1807, mas somente em 1820 notou que a corrente elétrica que passam num fio podia deflexionar uma agulha imantada. Neste mesmo ano os franceses Jean
Baptiste Biot (1774 - 1862) e Félix Savart (1791

- 1841) apresentaram uma quantificação do eletromagnetismo. Em 1825 o também francês André Marie Ampère (1775 - 1836) observou que dois fios paralelos conduzindo correntes elétricas trocavam forças entre si e que um fio em espiral comportava-se como um imã. O inglês Michael Faraday (1791 - 1867), em 1831, observou o surgimento de uma corrente elétrica num fio condutor quando se desloca um imã próximo a ele e também que variando a corrente elétrica num fio podia produzir corrente elétrica num outro fio próximo (este é o conceito de indução eletromagnética), mas na mesma época o estadunidense Joseph Henry (1797 - 1878) também descobriu a indução eletromagnética chegando a até construir o primeiro motor elétrico. Em 1841, o britânico William Thomson $(1824-1907)$ foi o primeiro a dar um tratamento matemático ao conceito de linhas de força introduzido por Faraday, sendo que em 1849 apresentou um trabalho sem fazer uso de fluido magnético ou elétrico. Vale destacar que na época não se aceitava a ideia de ação à distância e sim da existência de um fluido elétrico e/ou magnético como meio de propagação (também denominado éter ${ }^{3}$ ). O britânico James Clerk Maxwell (1831 - 1879) calculou a velocidade de propagação das ondas eletromagnéticas e verificou que era igual à da luz. Foi ele quem criou o termo campo

${ }^{3}$ Éter, na época, era considerado como o meio material que permeava o espaço e que possibilitava a propagação da luz e de outras ondas eletromagnéticas (TIPLER e LLEWELLYN, 2001). 
magnético, mas acreditava na existência do éter. Escreveu o livro Tratado sobre eletricidade e eletromagnetismo em 1873 onde enunciou as equações de Maxwell.

Já Bergia (2012) destaca que as equações de Maxwell revelam a natureza eletromagnética da luz e o carácter fundamental da velocidade da luz no vácuo e em relação ao referencial éter, sendo que, em 1878, Maxwell calculou o tempo gasto pela luz para fazer uma ida e volta na direção do vento do éter, chegando à estimativa de $10^{-8} \mathrm{~s}$.

O holandês Hendrik Antoon Lorentz (1853 - 1928), em sua tese de doutorado defendida em 1875, utilizou as equações de Maxwell para explicar a relação entre a eletricidade, o magnetismo e a luz. Forneceu detalhes matemáticos sobre a reflexão e refração da luz. Postulou que os átomos eram constituídos de partículas carregadas e que as oscilações dessas cargas produziam a luz (PIRES, 2008).

Em 1894, o irlandês George Johnstone Stoney (1826 - 1911) sugeriu o nome de elétrons para essas partículas (TIPLER; LLEWELLYN, 2001).

Segundo Bergia (2012), o físico alemão Heinrich Rudolf Hertz (1857 - 1894), em 1886, demonstrou a existência das ondas eletromagnéticas ao evidenciar a sua ação à distância, ele inventou um oscilador que permitia criar correntes alternadas de alta frequência e observou que estas induziam correntes em um condutor localizado a alguns metros de distância, mas não esclareceu o modo de propagação.

Pires (2008) aponta que em 1886 Hertz mediu as velocidades das ondas eletromagnéticas chegando ao resultado de $2,88 \times 10^{8} \mathrm{~m} / \mathrm{s}$. Além disso, mostrou que ocorrem os fenômenos de reflexão, refração e interferência. A partir de 1894 essas ondas hertzianas foram utilizadas para telegrafia sem fio, tendo como pioneiro o inglês Oliver Joseph Lodge (1851 - 1940). O britânico John Joseph Thomson $(1856$ - 1940) mediu a relação entre a carga e a massa das partículas que compõem o átomo, conforme definido por Lorentz, usando um tubo de raios catódicos. Demonstrou que um campo magnético podia afetar a frequência da luz emitida.

Já Eisberg e Resnick (1979) descrevem que Hertz, em 1886 e 1887, confirmou a existência de ondas eletromagnéticas e a teoria Maxwell sobre a propagação da luz e descobriu que uma descarga elétrica entre dois eletrodos ocorre mais facilmente quando incide luz ultravioleta neles, mas não conseguiu explicar o motivo.

Segundo Tipler e Llewellyn (2001), em 1896 o holandês Pieter Zeemann (1865 - 1943) obteve provas da existência de cargas em partículas examinando a interferência na luz emitida por átomos na presença de um campo magnético, chegando à conclusão que aparece uma força adicional que altera a frequência da oscilação e que as partículas que emitiam luz possuíam carga negativa. 
Já Bergia (2012) destaca que Thomson descobriu em 1897 que os raios catódicos ${ }^{4}$ eram constituídos por partículas de carga negativa (futuramente chamados de elétrons, conforme proposto por Stoney) e que o modelo atômico ficou estabelecido, a partir desta descoberta, como uma esfera positiva com pequenas partículas negativas incrustadas nesta esfera. Calculou a relação da carga (q) e massa (m) dos elétrons.

Em 1900 o alemão Philipp Lenard (1862 1947) utilizou um equipamento (figura 1) para estudar a emissão das partículas pelos cátodos quando submetidas à luz, conforme observado primeiramente por Hertz, submetendo-as a um campo magnético. Descobriu que a razão entre a carga e a massa era muito semelhante à dos raios catódicos obtida por Thomson, chegando à conclusão que eram partículas negativas (sabese hoje que são elétrons e que esse fenômeno é chamado de efeito fotoelétrico), mas também não apresentou nenhuma teoria satisfatória sobre essa observação (TIPLER;

\section{LLEWELLYN, 2001)}

$\mathrm{Na}$ figura 1 é possível verificar a representação esquemática do equipamento utilizado por Lenard. A luz proveniente da fonte $\mathrm{L}$ incide no cátodo C. As partículas

4 Raios catódicos são feixes de elétrons produzidos em um cátodo por causa de uma diferença de potencial existente entre esse cátodo e um ânodo no interior de um recipiente fechado contendo um gás rarefeito. Os físicos da época utilizaram muito este dispositivo para fazer experimentos sobre eletromagnetismo, sendo que o pioneiro foi o inglês William Crookes (1832 1919). emitidas pelo cátodo que passam pelo furo do ânodo A são indicadas em $\square$. Um campo magnético ente $A$ e $\square$ podia defletir as partículas para b, o que permitia determinar o sinal da carga das partículas e a razão $\mathrm{q} / \mathrm{m}$.

Figura 1: Representação do equipamento utilizado por Lenard

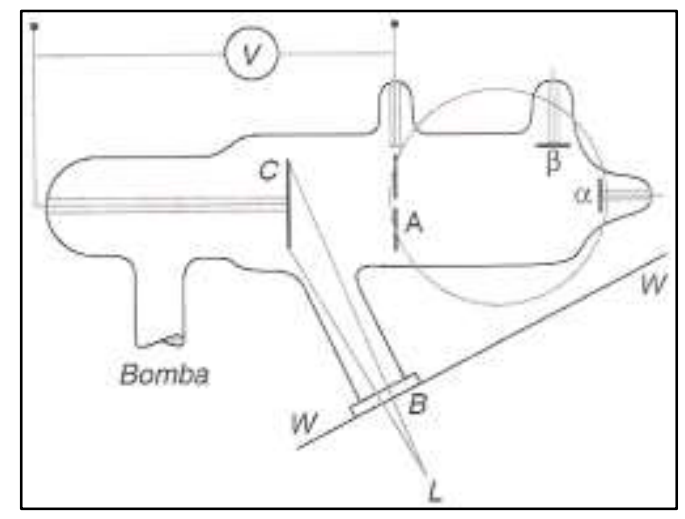

Fonte: Tipler e Llewellyn (2001, p.88)

Segundo Martins e Rosa (2014), os assistentes do alemão Max Von Loue (1879 1960), também alemães Walther Friedrich (1883 - 1968) e Paul Knipping (1883 - 1935), elaboraram um experimento onde um feixe de raios $\mathrm{X}$ atravessava um cristal e produzia um padrão de interferência, provando, em 1912, que os raios $\mathrm{X}$ eram ondas eletromagnéticas.

A evolução do eletromagnetismo, além de explicar os fenômenos elétricos e magnéticos, proporcionou um entendimento maior sobre a estrutura atômica, mas ainda havia muita coisa a ser explicada. 


\section{Nascimento da Física Quântica.}

Conforme Halliday, Resnick e Walker (2003) uma grandeza física que pode apresentar somente valores discretos (inteiros) é chamada de grandeza quantizada, já as grandezas físicas que podem apresentar qualquer valor numérico é chamada de grandeza contínua.

Segundo Gribbin (2002) a física quântica nasceu da necessidade de explicar a irradiação de luz por um objeto aquecido, sendo a luz considerada como onda (sabe-se hoje que isso ocorre devido à vibração dos elétrons).

Pires (2008) destaca que o alemão Max Planck (1858 - 1947) é considerado o pioneiro da Física Quântica devido à publicação de seu artigo em 1901 sobre o espectro da radiação emitida por um corpo negro. Ele propôs que osciladores harmônicos em equilíbrio com a radiação podiam absorver e emitir somente em quantidade discreta com a energia $(\varepsilon)$ de cada pacote que ele denominou de quanta, mas não explicou o fenômeno, utilizou esse conceito somente para correção e não estendeu a ideia de quantização para radiação eletromagnética.

Martins e Rosa (2014) apontam que Planck não tinha a intenção de quantizar a energia, ele supôs que a energia deveria ser dividida em um número definido de partes e introduziu a ideia de energia mínima através da equação 6, mas não enunciou que a energia total era um múltiplo inteiro dessa equação, tanto que em suas deduções matemáticas era sempre utilizada a teoria eletromagnética clássica, onde emissão e absorção são contínuas, inclusive declarou que a equação 6 surgiu como um ato de desespero para explicar os resultados experimentais.

$$
\varepsilon=h . f
$$

Onde:

$\mathrm{h}=$ constante de Plank $=6,63 \times 10^{-34} \mathrm{Js}$;

$\mathrm{f}=$ frequência;

Bergia (2012) enfatiza que Planck era um defensor da natureza ondulatória da luz e que nunca aceitou a teoria da quantização da radiação.

Conforme Martins e Rosa (2014), Lenard, em 1902, mostrou que a velocidade máxima das partículas chamadas catódicas era independente da intensidade luminosa, mas dependia da natureza da luz (frequência). Já em 1905, o físico alemão Albert Einstein (1879 - 1955) publicou um artigo onde apontava que a teoria ondulatória da luz tem um domínio limitado, pois a luz emitida consistia de um número finito de quanta de energia que podem ser produzidos e absorvidos como unidades completas. Provou matematicamente que a lei de Rayleigh- Jeans tinha um erro grave, pois descrevia uma densidade de energia infinita quando integrada sobre todas as frequências. Já Tipler e Llewellyn (2001) destacam que Einstein propôs que a luz é constituída por quanta de energia com valor igual ao produto hf, em vez de estar distribuída uniformemente no espaço. 
Eisberg e Resnick (1979) destacam que a interação da luz com a matéria pode causar a emissão de partículas negativas (efeito fotoelétrico) e que há três aspectos principais que não podem ser explicados com a teoria ondulatória clássica da luz:

a) $\mathrm{O}$ efeito fotoelétrico não ocorre para qualquer frequência de luz, independentemente de sua intensidade, contradizendo a teoria ondulatória que aponta que se a luz tiver intensidade suficiente, qualquer frequência ocasionaria o efeito da ejeção de partículas negativas (elétrons).

b) A emissão das partículas negativas é instantânea, não ocorre retardamento, e não importa a intensidade da luz, fato esse não explicado pela teoria ondulatória, pois a energia é distribuída uniformemente sobre a frente de onda e levaria um intervalo de tempo para a partícula absorver essa energia quando a luz for de pouca intensidade.

c) A energia cinética $(\mathrm{K})$ das partículas negativas emitidas não aumenta com o aumento da intensidade da luz, ela não ultrapassa o valor máximo dado pelo produto da carga elementar (e) pelo potencial limite ou de corte $\left(\mathrm{V}_{\mathrm{o}}\right)$, conforme equação 7 , isto é, o potencial limite tem sempre o mesmo valor para cada material independente da intensidade da luz.

$$
K_{\text {máx }}=e \cdot V_{o}
$$

Tipler e Llewellyn (2001) destacam a imposição de Einstein de que a energia cinética máxima também pode ser calculada através da frequência (f), da constante de Planck (h) e da função trabalho $(\phi)$, função essa que representa a energia necessária para remover um elétron da superfície (equação 8).

$$
K_{\text {máx }}=h . f-\phi
$$

Ainda em 1905, Einstein explicou o movimento browniano ${ }^{5}$ dizendo que os movimentos irregulares eram colisões das partículas suspensas com as moléculas dos líquidos, pois pelo tamanho reduzido e pelas massas pequenas das partículas suspensas, as colisões com as moléculas possuem efeitos consideráveis, mas a distância entre as colisões são "grandes" (microscopicamente) e com isso forma uma trajetória irregular e observável (PIRES, 2008).

Martins e Rosa (2014) apontam que em 1906 Einstein publicou outro artigo deixando claro a sua oposição a Planck com relação a quantização da radiação, mas utilizava como base a ideia da energia mínima de Plank para apoiar sua teoria de quanta de luz. Em 1907, no seu terceiro artigo sobre a quantização, Einstein desenvolveu a teoria do calor específico dos

5 Movimento em forma de ziguezague de partículas, como pólen ou fuligem, quando imersas em um líquido. Foi observado pela primeira vez em 1827 pelo escocês Robert Brown (1773 - 1858) com o auxílio de um microscópio. 
sólidos através da teoria de Planck e também chegou à quantização da energia de vibração atômica sem relacionar a quantização da luz.

Segundo Martins e Rosa (2014), o britânico Joseph John Thomson (1856 - 1940), em 1907, utilizou o conceito de frentes de ondas descontínuas para explicar o efeito fotoelétrico da radiação ultravioleta, destacando que a energia cresce com a frequência e que a radiação intensa se comporta quase como contínua e a pouco intensa possui uma natureza granular notável, principalmente a de alta frequência. Não discutiu sobre a radiação do corpo negro. Ainda em 1907, o alemão Johannes Stark (1874 - 1957), estudando a produção de raios $\mathrm{X}$ e a emissão de elétrons sob a ação de raios $\mathrm{X}$, supôs que a radiação se propaga pelo espaço sob a forma de quanta de energia. Em 1909 anunciou apoio à ideia de quanta de luz e que eles possuem quantidade de movimento (p) dada pela equação 9. Neste mesmo ano Einstein publicou dois artigos sobre a natureza da radiação e neles afirmava que a fórmula de Planck do corpo negro tinha uma relação direta com os quanta de luz.

$$
p=\frac{h f}{c}
$$

Sendo c a velocidade luz.

\subsection{A evolução do modelo da estrutura atômica como contribuição à física quântica.}

No início do século XIX, o inglês John Dalton (1766 - 1844) enunciou que a matéria é constituída por partículas fundamentais, em forma de esfera, maciça, homogênea, indivisível e indestrutível e com carga nula denominada de átomos. Somente em 1897, conforme já mencionado, é que Thomson, com base em seus experimentos sobre raios catódicos, propôs que: "Um átomo seria uma esfera uniforme de eletricidade positiva, de mais ou menos $10^{-8} \mathrm{~cm}$ de raio, com elétrons incrustados nesta esfera, de maneira a fornecer o arranjo eletrostático mais estável" (MAHAN, 1972, p. 307).

O neozelandês Ernest Rutherford (1871 1937), juntamente com seus alunos o alemão Johannes Wilhelm Geiger (1882 - 1945) e o inglês Ernest Marsden (1889 - 1970), realizando experimentos com partículas alfa incidindo numa folha de ouro observaram em 1909 desvios significativos dessas partículas inclusive delas retornando no sentido oposto à da incidência. Com isso, em 1910, tiveram a ideia de um átomo com uma carga positiva e massa concentrada numa região central (núcleo) muito inferior a nuvem de partículas negativas (elétrons) que o circundam (TIPLER; LLEWELLYN, 2001).

Em 1911, após quatro anos de experimentos e teorias, o físico estadunidense Robert Andrews Millikan (1868 - 1953) encontrou a carga elétrica do elétron $\left(1,6 \times 10^{-19}\right.$ C) através do famoso experimento da gota de óleo.

Em 1913 o dinamarquês Niels Henrick David Bohr (1885 - 1962) publicou um 
trabalho sobre um novo modelo atômico tendo os elétrons movendo-se em órbitas préestabelecidas, isto é, proibindo que os elétrons orbitem posições intermediárias, tendo a emissão de uma determinada radiação por parte do átomo quando um de seus elétrons mudasse de órbita (PIRES, 2008).

Martins e Rosa (2014) apontam que nessa publicação Bohr mostrou a quantização para o movimento dos elétrons em órbitas estáveis através da equação 10.

$$
m v R=\frac{n h}{2 \pi}
$$

Onde:

$\mathrm{m}=$ massa.

$\mathrm{v}=$ velocidade

$\mathrm{R}=$ raio da órbita.

$\mathrm{n}=$ número quântico principal.

Já Tipler e Llewellyn (2001) ressalta que o modelo de Bohr permitia prever com precisão as posições das linhas do espectro do átomo de hidrogênio, mas encontrou um problema em relação às consolidadas leis do eletromagnetismo que consideram que toda carga acelerada irradia uma onda eletromagnética e como o elétron possui uma aceleração centrípeta, pois se encontra numa trajetória circular, ele deveria emitir essa radiação e consequentemente com essa perda de energia iria diminuir seu raio orbital espiralando para o núcleo, fato esse que já se sabia na época que não ocorria. Assim, Bohr teve que enunciar três postulados: o primeiro foi que os elétrons se movem em suas órbitas sem irradiar energia, o segundo que os átomos irradiam quando um elétron sofre uma transição de um estado estacionário para outro e a frequência da radiação está relacionada com as energias das órbitas no estado inicial $\left(\mathrm{E}_{\mathrm{i}}\right)$ e no estado final $\left(\mathrm{E}_{\mathrm{f}}\right)$ através da equação 11 (chamado de condição de frequência) e o terceiro onde apontava que no limite de grandes órbitas e altas energias, os resultados quânticos devem coincidir com os resultados clássicos (chamado de princípio da correspondência).

$$
h f=E_{i}-E_{f}
$$

O físico inglês Henry Gwyn Jeffreys Moseley (1887 - 1915), em 1913, mediu os espectros de raios $\mathrm{X}$ emitidos por aproximadamente 40 elementos químicos diferentes e observou que as posições das linhas dos espectros variavam de forma regular, diferente do que acontecia no espectro óptico, chegando à conclusão de que isso ocorria devido a transições de elétrons situados nas órbitas próximas ao núcleo atômico, comprovando nessas transições que o átomo irradia uma energia específica conforme previsto por Bohr. Em 1914, os alemães James Frank (1882 - 1964) e Gustav Ludwin Hertz (1887 - 1975) realizaram o famoso experimento de Franck-Hertz que verificava a energia de 
elétrons ejetados de um cátodo aquecido após colidir com os elétrons de um gás no interior de um tubo de raios catódicos. Os resultados confirmaram a hipótese de Bohr de que os níveis de energia dos átomos são quantizados. Essa técnica foi melhorada e hoje se tem uma forma muito precisa de medirem os estados quantizados dos átomos em gases e sólidos chamado de espectroscopia por perda de energia de elétrons (TIPLER; LLEWELLYN, 2001).

Entre 1914 e 1915, o alemão Arnold Johannes Wilhelm Sommerfeld (1868 a 1951) propôs a inclusão de órbitas elípticas ao invés das órbitas circulares propostas por Bohr e também o trabalho com elétrons tendo altas velocidades (alta energia), incluindo para isso a relatividade restrita de Einstein, isto é, ele propôs uma generalização da teoria de Bohr. Ainda em 1914, Stark, que havia anunciado em 1909 apoio a teoria dos quanta, mudou de ideia quando observou linhas espectrais emitidas pelos raios $\mathrm{X}$ e Rutherford et. al., medindo a velocidade dos elétrons emitidos quando submetidos a raios $\mathrm{X}$, confirmaram a relação $\mathrm{E}=$ h.f (MARTINS; ROSA, 2014).

\subsection{A confirmação da teoria dos quanta de Einstein.}

Entre os anos de 1910 e 1911 pouco se discutia sobre a teoria da radiação e os quanta, inclusive na famosa conferência de Solvay, em 1911, quase não se comentou no assunto, até Einstein resolveu manter-se em silêncio.
Em 1912, Max Planck definiu que a energia eletromagnética era distribuída continuamente no espaço, assim como a absorção dessa energia pelos osciladores, mas a emissão seria descontínua, ocorrendo quando atingisse uma energia múltipla do produto h.f. Neste mesmo ano, Friedrich e Knipping, como já mencionado, provaram que os raios $\mathrm{X}$ são ondas eletromagnéticas, mas mesmo assim, o físico britânico William Henry Bragg (1862 1942) afirmava que tanto os raios $X$ como a luz tinham propriedades corpusculares e ondulatórias (MARTINS; ROSA, 2014).

Conforme Tipler e Llewellyn (2001), entre 1914 e 1916, Millikan realizou uma série de experimentos relacionando o potencial de corte da corrente elétrica no efeito fotoelétrico com a frequência emitida, obtendo gráficos com retas cuja inclinação é dada pela razão da constante de Plank (h) pela carga elétrica do elétron (e), conforme previsto por Einstein na junção das equações 7 e 8 . A figura 2 apresenta um gráfico apontando o resultado obtido por Millikan em 1915, que comprova a veracidade da equação de Einstein para o efeito fotoelétrico, onde a inclinação da reta é a razão h/e. 
Figura 2: Resultados obtidos por Millikan e plotados num gráfico do potencial de corte em função da frequência.

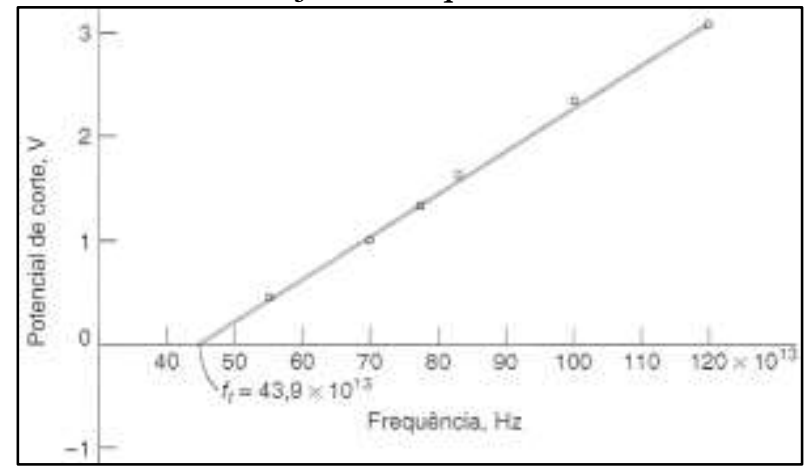

Fonte: Tipler e Llewellyn (2001, p.89).

Gribbin (2002) ressalta que Millikan passou 10 anos de sua vida realizando experimentos em busca de provar que Einstein estava errado, mas ao final das contas acabou provando o contrário, isto é, que Einstein estava certo.

Em 1916, Einstein publica um artigo definindo a propriedade corpuscular da luz, confirmando que sua energia é dada pela equação 6 (já definida por Plank em 1901, mas somente para emissão), assim como que a quantidade de movimento é dada pela equação 9 (já definida por Stark em 1909). Em 1921, Einstein recebe o prêmio Nobel pela sua contribuição à física teórica sobre o efeito fotoelétrico e em 1923 Millikan também foi agraciado com o prêmio Nobel por seus trabalhos sobre a carga elétrica elementar e o efeito fotoelétrico (BERGIA, 2012).

Em 1922, o estadunidense Arthur Holly Compton (1892 - 1962) elaborou uma experiência que comprova o caráter corpuscular devido ao espalhamento da radiação eletromagnética, conforme figura 3 (BERGIA, 2012).

Figura 3: Esquema da experiência de Compton.

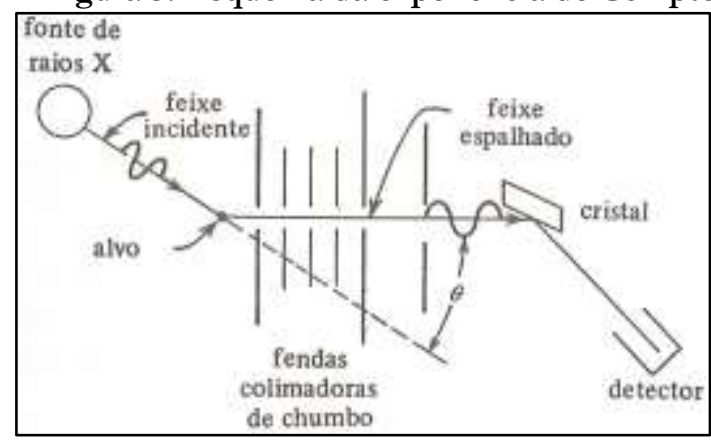

Fonte: Eisberg e Resnick (1979, p. 59).

Já Eisberg e Resnick (1979, p. 59 - 60) apontam que essa confirmação só foi alcançada em 1923 e descrevem em mais detalhes o efeito Compton:

A natureza corpuscular da radiação foi
dramaticamente confirmada em 1923 pelas
experiências de Compton. Ele fez com que um feixe
de raios $X$ de comprimento de onda $\lambda$ incidisse sobre
um alvo de grafite, como é mostrado na figura [...].
Mediu-se a intensidade dos raios X espalhados em
função de seu comprimento de onda, para vários
angulos de espalhamento. [...] embora o feixe
incidente consista essencialmente de um único
comprimento de onda $\lambda$, os raios X espalhados têm
máximos de intensidade em dois comprimentos de
onda; um deles é o mesmo que o comprimento de
onda incidente, e o outro $\lambda$ ', é maior que $\lambda$ [...].
A presença do comprimento de onda $\lambda$ não pode ser
compreendida se os raios X incidentes forem
encarados como uma onda eletromagnética clássica.
No modelo clássico o campo elétrico oscilante com
frequência da onda incidente age sobre os elétrons
livres do alvo fazendo-os oscilar com a mesma
frequência. Esses elétrons, como cargas oscilando em
uma pequena antena de rádio, irradiam ondas
eletromagnéticas com a mesma frequência. Portanto,
no modelo clássico a onda espalhada deveria ter a
mesma frequência e o mesmo comprimento de onda $\lambda$
da onda incidente.
Compton (e independentemente Debye ${ }^{6}$ ) interpretou
seus resultados experimentais postulando que o feixe
de raios X incidente não era uma onda de frequência
$f$, mas um conjunto de fótons, cada um com energia

6 Petrus Josephus Wilhelmus Debye (1884 1966), físico-químico holandês. 
$E=h f$, e que esses fótons colidiam com os elétrons livres do alvo da mesma forma que colidem duas bolas de bilhar.

Já Martins e Rosa (2014) apontam que o efeito Compton foi muito importante para dar credibilidade à quantização da radiação, mas que ainda nada havia sido provado, inclusive Compton nunca citou os trabalhos de Einstein e Bohr criticou o trabalho de Compton alegando problemas com o fenômeno da interferência. Já Silva e Freire Jr (2014) destacam que o estadunidense William Duane (1972 - 1935) foi quem ofereceu uma grande resistência ao trabalho de Compton, mas que após alguns debates e trocas de informações sobre os experimentos Duane, através de experimentos, confirmou os resultados de Compton.

\section{$4.3 \mathrm{O}$ caráter ondulatório da matéria.}

O físico francês Louis de Broglie (1882 1987), em 1923, durante o desenvolvimento de sua tese de doutorado, sugeriu várias ideias, ainda de forma especulativa, sobre as partículas terem caráter ondulatório, assim como as teorias da época sugerirem a radiação eletromagnética terem comportamento de partícula (NUSSENZVEIG, 2002).

Já Martins e Rosa (2014) destacam que em 1923 de Broglie publicou três artigos, sendo que no primeiro suas principais ideias foram: não existe diferença entre os quanta e qualquer outra partícula, focou em deduções aplicando a relatividade de Einstein, as partículas possuem velocidades inferiores a da radiação eletromagnética e estão sujeitas a oscilações, toda partícula possui uma onda associada onde suas oscilações estão sempre em fase fazendo com que numa trajetória fechada (como órbitas dos elétrons) o movimento se torne estável. No segundo artigo ele destacou que todas as partículas podem sofrer difração, que a probabilidade de interação entre partícula e matéria é proporcional à intensidade da onda associada à partícula em cada posição do espaço, não obedecem à lei da inércia, se movem de acordo com raios perpendiculares às frentes de onda das ondas que estão em fase, as velocidades das partículas equivalem à velocidade de um conjunto de ondas de fases com frequências próximas e as ondas são capazes de guiar as partículas e determinar a probabilidade de que eles fossem absorvidos ou emitidos. Já no terceiro artigo ele analisou sobre a radiação do corpo negro e a estatística quântica. Defendeu sua tese em 1924, sem incluir nada relevante com relação ao que havia publicado nos artigos.

Os efeitos ondulatórios associados aos elétrons foram descobertos pela primeira vez em 1927 pelos físicos estadunidenses Clinton Joseph Davisson (1881 - 1958) e Lester Halbert Germer (1896 - 1971) através de um experimento com elétrons de baixa energia (40 a $68 \mathrm{eV}$ ) que apresentaram o fenômeno ondulatório de difração ao serem incididos em um cristal de níquel e detectados em uma câmara de ionização. Já para os átomos e moléculas a propriedade ondulatória de 
difração foi comprovada pela primeira vez em 1930 pelos físicos Stern e Estermann incidindo feixes de átomos de hélio e moléculas de hidrogênio em um cristal de fluoreto de lítio (TIPLER; LLEWELLYN, 2001).

\subsection{Problemas encontrados pela teoria quântica da época.}

A teoria quântica até o início da década de 1920 apresentou-se muito bem sucedida, mas ainda sujeita a algumas críticas, como o tratamento exclusivo a sistemas periódicos, a teoria atômica de Bohr que serve apenas para o átomo de hidrogênio, podendo ser estendida somente para os elementos alcalinos $(\mathrm{Li}, \mathrm{Na}, \mathrm{K}$, $\mathrm{Rb}$ e $\mathrm{Cs}$ ) por terem similaridades a um átomo de um elétron, a falta de previsão da determinação das intensidades das linhas espectrais e da probabilidade das ocorrências das transições dos elétrons entre os possíveis estados de energia e da teoria apresentar alguma incoerência com relação aos conceitos físicos (EISBERG; RESNICK, 1979).

Surge então a necessidade de um desenvolvimento teórico mais preciso e de fundamentações matemáticas mais sofisticadas para consolidar a teoria quântica. Essa época marca a transição da chamada antiga teoria quântica e da nova teórica quântica denominada mecânica quântica ou mecânica ondulatória.

\section{Mecânica quântica.}

De acordo com Tipler e Llewellyn (2001), em 1924 o físico indiano Satyendra Nath Bose (1894 - 1974) desenvolveu uma teoria, com o auxílio de Einstein, sobre a distribuição estatística para os fótons e que se estendeu para todos os bósons ${ }^{7}$. Chegaram a uma distribuição conhecida por distribuição de Bose-Eisntein.

Em 1925, o alemão Werner Karl Heinsenberg (1901 - 1976) apresentou num artigo uma teoria matemática do átomo sem utilização de conceitos das posições dos elétrons, baseada em quantidades observadas em experimentos com as frequências das amplitudes das linhas de espectros (PIRES, 2008).

Em 1926, o austríaco Erwin Rudolf Josef Alexander Schrödinger (1887 - 1961) publicou um artigo onde continha uma equação que governa o movimento dos elétrons e outras partículas, que é análogo à equação de ondas da física clássica, pois relaciona as derivadas da função de onda $(\Psi)$ em relação ao tempo $(t)$ e em relação ao espaço (x), que para uma dimensão está representado pela equação 12. As teorias de Heisenberg e Schrödinger são equivalentes, tanto que o próprio Schrödinger provou que uma pode ser demonstrada a partir da outra. Essa teoria é uma das mais bem sucedidas da Física e é a base da mecânica

\footnotetext{
7 Partículas com spin inteiro ou nulo, como os fótons, glúons e mésons.
} 
ondulatória quântica (TIPLER; LLEWELLYN, 2001).

$$
-\frac{\hbar^{2}}{2 m} \frac{\partial^{2} \Psi_{(\mathrm{x}, \mathrm{t})}}{\partial x^{2}}+V(x, t) \Psi_{(\mathrm{x}, \mathrm{t})=\mathrm{i}} \hbar \frac{\partial \Psi_{(\mathrm{x}, \mathrm{t})}}{\partial_{\mathrm{t}}}
$$

Onde:

$\hbar=$ Constante de Planck normalizada $\left(1,054 \cdot 10^{-34} \mathrm{~J} \mathrm{~s}\right)$.

$\mathrm{V}=$ Energia potencial.

Segundo Pires (2008), em 1926, o físico matemático alemão Max Born (1882 - 1970) propôs uma interpretação probabilística da função de onda de Schrödinger demonstrando que o quadrado da função de onda representa a densidade de probabilidade de encontrar uma partícula num determinado ponto (equação 13). Ele é considerado o pioneiro da visão probabilística na mecânica quântica.

$$
P(x) \propto|\Psi|^{2}
$$

Já Bergia (2012) comenta que a definição de Born é que as ondas nada mais são do que a expressão probabilística da posição das partículas e que em 1927 foi enunciado o princípio da incerteza onde não é possível determinar com precisão a localização $(\mathrm{x})$ e a quantidade de movimento (p) de uma partícula ao mesmo, sendo que o produto das incertezas da posição e da quantidade de movimento não pode ser inferior à constante de Planck $h$ (equação 14).

$$
\Delta x . \Delta p \geq h
$$

Para rebater às criticas de Einstein ao princípio das incertezas, Bohr provou que o produto das incertezas da energia e do tempo também não pode ser inferior à constante de Planck, (equação 15). Além disso, Bohr enunciou o conceito de complementariedade, onde determina que os objetos quânticos são ondas ou corpúsculos e também ondas e corpúsculos, isto é, eles são complementares (BERGIA, 2012).

$$
\Delta E . \Delta t \geq h
$$

Born, Bohr, Heisenberg, o alemão Ernest Pascual Jordan (1902 - 1980), o austríaco Wolfgang Ernst Pauli (1900 - 1958) e o britânico Paul Adrien Maurice Dirac (1902 1984) desenvolveram a probabilidade e a ondulatória para a mecânica quântica (PIRES, 2008).

Em 1928, Dirac apresentou uma equação relativística para o elétron que previa spin 1/2, destacou também a teoria da existência de uma antimatéria que podia aniquilar a matéria, destacando a existência de uma antipartícula (pósitron) que quando ocorresse o encontro com a partícula (elétron) ocorreria a 
aniquilação. Em 1932, o estadunidense Carl David Anderson (1905 - 1991) descobriu o pósitron experimentalmente, afirmando que ele possui a mesma massa e spin do elétron, mas carga elétrica oposta (PIRES, 2008).

Dirac com a ajuda do físico italiano Enrico Fermi (1901 - 1954) complementaram a estatística quântica determinando a função de distribuição para os férmions ${ }^{8}$ que foi denominada de distribuição de Fermi-Dirac (TIPLER; LLEWELLYN, 2001).

Até 1932, as equações e conceitos físicos, assim como a concordância com os resultados experimentais, estavam de acordo, mas as interpretações dos físicos eram diversas, dentre elas cinco alcançaram destaque, conforme exposto no quadro 1 (MARTINS; ROSA, 2014).

${ }^{8}$ Partículas com spin fracionário como os elétrons, prótons e nêutrons. Obedecem ao princípio de exclusão de Pauli, isto é, dois férmions idênticos não podem ocupar o mesmo estado quântico simultaneamente. 
Quadro 1: Teorias principais sobre as interpretações dos fenômenos quânticos

\begin{tabular}{|l|l|}
\hline \multicolumn{1}{|c|}{ Teoria } & \multicolumn{1}{c|}{ Descrição } \\
\hline Interpretação de & $\begin{array}{l}\text { Defendidas por Born, Bohr, Heisenberg, Jordan, Pauli e Dirac, que unificaram noções } \\
\text { probabilísticas e ondulatórias. Definiram que entidades quânticas se comportam algumas } \\
\text { vezes como partículas e outras como ondas. }\end{array}$ \\
\hline $\begin{array}{l}\text { Interpretação } \\
\text { Semiclássica }\end{array}$ & $\begin{array}{l}\text { Defendidas por de Broglie, Schrödinger e Einstein. Definem que uma partícula é } \\
\text { acompanhada por uma onda e o movimento da partícula é influenciado por essa onda. }\end{array}$ \\
\hline Colapso da função onda & $\begin{array}{l}\text { Proposto pelo estadunidense Wojciech Hubert Zurek (nascido em 1951). Define que o } \\
\text { ambiente atua como um aparato para monitorar o sistema, isto é, o ambiente define a } \\
\text { fronteira entre fenômenos clássicos e quânticos (decoerência quântica). }\end{array}$ \\
\hline Muitos universos & $\begin{array}{l}\text { Proposto pelo físico estadunidense Hugh Everett (1930 - 1982). Cada estado quântico é } \\
\text { uma realidade física separada. Nunca ocorre colapso da onda, pois o universo se divide em } \\
\text { cópias incontáveis e cada mundo possui o seu próprio espaço-tempo. }\end{array}$ \\
\hline Variáveis ocultas & $\begin{array}{l}\text { Desenvolvida pelo irlandês John Stewart Bell (1928 - 1990) e pelo estadunidense David } \\
\text { Joseph Bohm (1917 - 1992). Tinha como base que o estado físico é definido por variáveis } \\
\text { ocultas não observáveis, onde ainda não se tem a habilidade de determinar com precisão } \\
\text { esses parâmetros ocultos. }\end{array}$ \\
\hline
\end{tabular}

Fonte: Elaborado pelo autor.

Por incrível que pareça, todas essas teorias possuem uma explicação física consistente e são demonstradas partindo das mesmas formulações matemáticas.

Em 1935, Albert Einstein, juntamente com o ucraniano Boris Podolsky (1896 - 1966) e o estadunidense Nathan Rosen (1909 - 1995) publicou um artigo onde afirmava que a mecânica quântica defendida pela interpretação de Copenhague era incompleta, pois a mesma defendia a não localidade, isto é, mesmo que duas partículas que sejam correlacionadas inicialmente se afastarem de maneira que não se comuniquem, elas não são consideradas como partes separadas, uma delas poderia influenciar na outra independente da distância e de maneira instantânea (PESSOA, 2006).
Em 1952, Bohm apresentou uma teoria, apoiado nos trabalhos de De Broglie, sobre a teoria das variáveis ocultas e de carácter não local, bastante consistente, mas que não foi aceita a princípio pela comunidade científica (PESSOA, 2006).

Segundo Bergia (2012), o físico Bell publicou em 1964 um teorema, chamado teorema de Bell ou desigualdade de Bell, onde propunha um experimento para verificar se o ponto de vista do realismo local de Einstein estava correto ou se o ponto de vista da não localidade da mecânica quântica de Bohr é que estava correto. Em 1969 o físico estadunidense John Francis Clauser (nascido em 1942) et al projetaram um dispositivo para testar o teorema de Bell e os resultados ficaram a favor da mecânica quântica de Bohr, mas o dispositivo 
ainda não era confiável. Somente em 1982 que o francês Alain Aspect (nascido em 1947) et. al. elaboraram uma experiência mais precisa e novamente os resultados foram a favor da mecânica quântica de Bohr. Em 1988 um maior aperfeiçoamento do experimento ocorreu por parte do austríaco Anton Zeiling (nascido em 1945) et. al. e mais uma vez concordou com as previsões da mecânica quântica de Bohr.

Mesmo com os resultados experimentais apontando para uma teoria não local, a única das cinco teorias apontadas que é afetada na sua essência é a da interpretação semiclássica, as outras teorias podem ser tanto de carácter local como de não local, mas isso não descredencia a interpretação semiclássica, pois além da possibilidade de readaptá-la a esses resultados, os experimentos e teorias sobre o assunto se multiplicam a cada dia e muitas informações ainda estão por vir.

\section{Conclusão}

Neste artigo foi demonstrada a evolução da Física Quântica apontando as principais etapas para o seu surgimento e desenvolvimento, mas procurando utilizar uma linguagem que não necessite de conhecimentos aprofundados em Física ou Matemática para a sua leitura, sendo atingido o propósito de proporcionar uma visão de como as teorias e experimentos surgiram para o desenvolvimento da Física Quântica.

Vale destacar que vários ramos da Física foram aperfeiçoados após a consolidação da
Física Quântica como a Física Nuclear, Física Atômica, Física do Estado Sólido, Teoria Quântica dos Campos, Eletrodinâmica Quântica, Cromodinâmica Quântica, Física das Partículas, Astrofísica e Cosmologia. Além disso, a Física Quântica é utilizada em diversos ramos da ciência, como na Engenharia, Medicina, Ciências Biológicas, Química entre outros.

Existem alguns pontos ainda divergentes na comunidade científica e por esse motivo há teorias diferentes na Física Quântica, mas com o desenvolvimento tecnológico, os instrumentos e dispositivos utilizados nos experimentos se tornam mais precisos a cada dia, e é muito provável que muitas descobertas surgirão nos próximos anos.

\section{Referências bibliográficas}

BERGIA, Silvio. Gênios da Ciência: Einstein. Scientific American Brasil. 2 ed. São Paulo: Duetto Editorial, p. 5-98, 2012.

EISBERG, Robert; RESNICK, Robert. Física Quântica. 23 ed. Rio de Janeiro: Elsevier, 1979.

GRIBBIN, John. Fique por dentro da Física Moderna. 2 ed. São Paulo: Cosac e Naify, 2002.

HALLIDAY, David; RESNICK, Robert; WALKER, Jearl. Fundamentos de Física: 
Gravitação, ondas e termodinâmica. 6. ed. Rio de Janeiro: LTC, 2002. v2.

HALLIDAY, David; RESNICK, Robert; WALKER, Jearl. Fundamentos de Física: Eletromagnetismo. 6. ed. Rio de Janeiro: LTC, 2003. v3.

MAHAN, Bruce H. Química: um curso universitário. 2. ed. São Paulo: Edgard Blücher, 1972.

PESSOA, Osvaldo J. Conceitos de Física Quântica. São Paulo: Livraria da Física, 2006. v2.

PIRES, Antonio S. T.. Evolução das ideias da Física. São Paulo: Livraria da Física, 2008.

SILVA, Indianara; FREIRE Jr, Olival. A descoberta do efeito Compton: De uma abordagem semiclássica a uma abordagem quântica. Revista Brasileira do Ensino de Física 36, n. 1, 1601 (2014).

TIPLER, Paul A.; LLEWELLYN, Ralph A. Física Moderna. 3 ed. Rio de Janeiro: LTC, 2001.

MARTINS, Roberto de Andrade; ROSA, Pedro Sérgio. História da teoria quântica: a dualidade onda-partícula, de Einstein a De Broglie. São Paulo: Livraria da Física, 2014.

NUSSENZVEIG, H. Moysés. Curso de Física Básica: Ótica, Relatividade e Física
Quântica. 1 ed. São Paulo: Edgard Blücher, 2002. V4. 